\title{
Synthetic X-ray light curves of BL Lacs from relativistic hydrodynamic simulations
}

\author{
P. Mimica ${ }^{1}$, M. A. Aloy ${ }^{1}$, E. Müller ${ }^{1}$, and W. Brinkmann ${ }^{2}$ \\ 1 Max-Planck-Institut für Astropysik, Postfach 1312, 85741 Garching, Germany \\ 2 Max-Planck-Institut für extraterrestrische Physik, Postfach 1603, 85740 Garching, Germany
}

Received 2 September 2003 / Accepted 12 February 2004

\begin{abstract}
We present the results of relativistic hydrodynamic simulations of the collision of two dense shells in a uniform external medium, as envisaged in the internal shock model for BL Lac jets. The non-thermal radiation produced by highly energetic electrons injected at the relativistic shocks is computed following their temporal and spatial evolution. The acceleration of electrons at the relativistic shocks is parametrized using two different models and the corresponding X-ray light curves are computed. We find that the interaction time scale of the two shells is influenced by an interaction with the external medium. For the chosen parameter sets, the efficiency of the collision in converting dissipated kinetic energy into the observed X-ray radiation is of the order of one percent.
\end{abstract}

Key words. galaxies: BL Lac objects: general X-rays: general galaxies: quasars: general - radiation mectranisms: non-thermal - acceleration of particles - methods: numerical

\section{Introduction}

BL Lac objects are thought to be dominated by relativistic jets seen at small angles to the line of sight (Urry \& Padovani 1995), and their remarkably featureless radio-through-Xray spectra are well fitted by inhomogeneous jet models (Bregman et al. 1987). As the measured spectra can be reproduced by models with widely different assumptions, the structure of the relativistic jets remains largely unknown. Only the analysis of the temporal variations of the emission and combined spectral and temporal information can considerably constrain the jet physics. Time scales of the observed light curves are related to the crossing time of the emission regions which depend on wavelength and/or the time scales of micro-physical processes like particle acceleration and radiative losses. The measured time lags between the light curves at different energies as well as spectral changes during intensity variations allow one to probe the microphysics of particle acceleration and radiation in the jet.

Recently, several extended observation campaigns on the prominent BL Lacs PKS 2155-304, Mrk 501, and Mrk 421 by ASCA and BeppoSAX, partly simultaneously with RXTE and $\mathrm{TeV}$ telescopes, have revealed that in general the X-ray spectral index and the peak energy correlate well with the source intensity (for a review see Pian 2002). The emission of the soft X-rays is generally well correlated with that of the

Send offprint requests to: $\mathrm{P}$. Mimica, e-mail: pere@mpa-garching.mpg.de hard X-rays and lags it by 3-4 ks (Takahashi et al. 1996, 2000; Zhang et al. 1999; Malizia et al. 2000; Kataoka et al. 2000; Fossati et al. 2000). However, significant lags of both signs were detected from several flares (Tanihata et al. 2001). From XMM-Newton observations of PKS 2155-304 Edelson et al. (2001) give, however, an upper limit to any time lags of $|\tau| \leq$ $0.3 \mathrm{~h}$. They suggest that previous claims of time lags of soft X-rays with time scales of hours might be an artifact of the periodic interruptions of the low-Earth orbits of the satellites every $\sim 1.6 \mathrm{~h}$. Large flares with time scales of $\sim 1$ day were detected with temporal lags of less than $1.5 \mathrm{~h}$ between X-ray and TeV energies (for Mrk 421 see Takahashi et al. 2000). For all three sources the structure function and the power density spectrum analysis indicate a roll-over with a time scale of the order of 1 day or longer (Kataoka et al. 2001) which seems to be the time scale of the successive flare events. On shorter time scales only small power in the variability is found with a steep slope of the power density spectrum $\sim f^{-(2-3)}$ (Tanihata 2002).

These results were obtained from data with a relatively low signal-to-noise ratio, integrated over wide time intervals (typically one satellite orbit). Uninterrupted data with high temporal and spectral resolution can now be provided by $X M M-N e w t o n$. From an analysis of early data taken with the XMM-Newton EPIC cameras from Mrk421, the brightest BL Lac object at X-ray and UV wavelengths, for the first time the evolution of intensity variations could be resolved on time scales of $\sim 100 \mathrm{~s}$ (Brinkmann et al. 2001). Temporal variations by a factor of three at highest $\mathrm{X}$-ray energies were 
accompanied by complex spectral variations with only a small time lag of $\tau=265_{-102}^{+116}$ s between the hard and soft photons.

In an extensive study of all currently available XMM-Newton observations of Mrk421 Brinkmann et al. (2003) find that the source exhibits a rather complex and irregular variability pattern - both, temporarily and spectrally. In general, an increase in flux is accompanied by a hardening of the spectrum as expected from a shift of the synchrotron peak to higher energies. But there are exceptions and the rate of the spectral changes varies strongly. The shortest variability time scales appear to be of the order of $\gtrsim k s$. The lags between the hard and soft band flux are small and can be of different sign.

Correspondingly, it is hard to deduce the underlying physical parameters for the radiation process from the observations. For the currently favored "shock-in-jet" model for the BL Lac emission (see, for example, Spada et al. 2001) this implies that we are seeing the emission from multiple shocks which have either widely different physical parameters or that we detect the emission from similar shocks at very different states of their evolution, additionally being masked by relativistic beaming and time dilatation effects.

The internal shock scenario assumes that an intermittently working central machine produces blobs of matter moving at different velocities along the jet. The interaction of two blobs is modeled as the collision of two shells whose interaction starts from the time of collision (Sikora et al. 2001; Spada et al. 2001; Moderski et al. 2003; Tanihata et al. 2003). This time is estimated from the relative velocity of two shells. During the interaction an internal shock propagates through the slower shell and accelerates electrons which produce the observed radiation (Spada et al. 2001; Bicknell \& Wagner 2002). These analytic models can be used to constrain the dimensions and physical properties of the emitting regions, but they cannot take into account the detailed hydrodynamic evolution of the interacting shells, nor the influence of the external medium prior to the interaction. To this end we have simulated the two dimensional axisymmetric evolution of two dense shells moving at different collinear velocities through a homogeneous external medium.

In Sect. 2 we describe the numerical method we have used to simulate both the hydrodynamics and the temporal evolution of non-thermal electrons in the fluid. The shock acceleration process is modeled by two different approaches which are described in detail in Sect. 3. The results of our study are presented and discussed in Sect. 4 , and the conclusions are given in Sect. 5.

\section{Numerical method}

We assume that the dynamics of blazars are dominated by the thermal (baryonic or cold) matter while their emission is produced by a non-thermal component, which is in agreement with Sikora et al. (2001). This is justified in our model because the number densities of electrons and protons are equal and, thus, the inertia of the baryons is much larger than that of the leptons.

We have performed a set of two dimensional axisymmetric simulations (in cylindrical coordinates $r$ and $z$ ) of dense shells of matter moving at different relativistic speeds in the same direction. The shells collide after some time giving rise to internal shocks, where part of the internal energy of the thermal fluid is transferred to relativistic electrons producing the observed synchrotron radiation.

The problem is split into two parts, a thermal and nonthermal one. The evolution of the thermal or hydrodynamic component of rest mass density $\rho$, pressure $P$, radial velocity $v_{\mathrm{r}}$ and axial velocity $v_{z}$ is simulated by means of a relativistic hydrodynamic code. The code also includes a set of $N$ additional fluid components tracing the evolution of non-thermal relativistic electrons at different energies. The tracer fluids of number density $n_{i}(i=1, \ldots, N)$ are advected by the thermal fluid, and are coupled in energy space by their radiative energy losses.

In the following subsections we detail the algorithms used for the simulation of the thermal fluid (Sect. 2.1), of the relativistic electrons (Sect. 2.2) and of the coupling between them (Sect. 2.4).

\subsection{Hydrodynamics}

The equations of relativistic hydrodynamics can be cast in a system of conservation laws of the form

$\frac{\partial \boldsymbol{U}}{\partial t}+\sum_{k=1}^{3} \frac{\partial \boldsymbol{F}^{i}}{\partial x_{i}}=0$

where $\boldsymbol{U}$ and $\boldsymbol{F}^{k}$ are the vector of conserved variables and the flux vectors, respectively (e.g., Marti et al. 1994). In the case of axial symmetry and expressed in cylindrical coordinates $(r, z)$ Eq. (1) reads

$\frac{\partial \boldsymbol{U}}{\partial t}+\frac{1}{r} \frac{\partial r \boldsymbol{F}}{\partial r}+\frac{\partial \boldsymbol{G}}{\partial z}=\boldsymbol{S}$

where

$\boldsymbol{U}=\left[\rho \Gamma, \rho h \Gamma^{2} v_{r}, \rho h \Gamma^{2} v_{z}, \rho h \Gamma^{2}-P-\rho \Gamma, \rho \boldsymbol{X} \Gamma\right]^{T}$

is the vector of conserved quantities and

$\boldsymbol{F}=\left[\rho \Gamma v_{r}, \rho h \Gamma^{2} v_{r}^{2}+P, \rho h \Gamma^{2} v_{z} v_{r}, \rho h \Gamma^{2} v_{r}-\rho \Gamma v_{r}, \rho \boldsymbol{X} \Gamma v_{r}\right]^{T}$

and

$\boldsymbol{G}=\left[\rho \Gamma v_{z}, \rho h \Gamma^{2} v_{r} v_{z}, \rho h \Gamma^{2} v_{z}^{2}+P, \rho h \Gamma^{2} v_{z}-\rho \Gamma v_{z}, \rho \boldsymbol{X} \Gamma v_{z}\right]^{T}$,

are the corresponding flux vectors in $r$ and $z$ direction, respectively. Note that for reasons of convenience the speed of light, which is denoted by $c$ elsewhere, is set equal to one in this subsection. Consequently, the Lorentz factor is given by $\Gamma \equiv\left(1-v_{r}^{2}-v_{z}^{2}\right)^{-1 / 2}$, and the specific enthalpy by $h=1+\varepsilon+P / \rho$, where $\varepsilon$ is the specific internal energy of the fluid.

$\boldsymbol{S}=\left[0, \frac{P}{r}, 0,0, \mathbf{0}\right]^{T}$

is the source vector expressing the non-conservation of the radial momentum in cylindrical coordinates, and

$\boldsymbol{X}=\left[X_{1}, \ldots, X_{N}\right]^{T}$,

is an $N$-component vector with

$X_{i}=\frac{n_{i} m_{\mathrm{e}}}{\rho}, i=1, \ldots, N$, 
where $m_{\mathrm{e}}$ is the electron rest mass, and $X_{i}$ is the mass fraction of the tracer species $i$ with respect to the mass of the thermal fluid.

The conservation laws are integrated with the GENESIS code of Aloy et al. (1999). This code, which exploits the piecewise parabolic method (Colella \& Woodward 1984), was suitably modified to passively advect a set of non-thermal species along with the main thermal fluid. We assume that the thermal component is a perfect fluid obeying an ideal equation of state of the form $P=\left(\gamma_{\mathrm{ad}}-1\right) \varepsilon \rho$, where $\gamma_{\mathrm{ad}}=4 / 3$ is the adiabatic index.

\subsection{Non-thermal population}

The non-thermal particle population evolves both in space and time. Limiting the physical conditions in the fluid in such a way that during a time step non-thermal particles are contained in a single numerical cell, it is possible to split the evolution of the non-thermal particles in space and in time. This implies that there is a minimum magnetic field or, equivalently, a maximum allowed Larmor radius that depends on the numerical resolution employed (see below). We point out, however, that there are other approaches which treat the evolution of nonthermal particles by solving the diffusion-convection equation (Miniati 2001; Jones et al. 1999). The spatial evolution of the non-thermal particles is done by advecting them along with the fluid and, thus, their macroscopic velocity field corresponds to that of the thermal fluid (see Sect. 2.1). The treatment of the temporal evolution is described in this section.

The non-thermal particle population is assumed to be composed of ultra-relativistic electrons injected at shocks. Its temporal evolution is governed by the kinetic equation (e.g., Kardashev 1962):

$\frac{\partial n(\gamma, t)}{\partial t}+\frac{\partial}{\partial \gamma}(\dot{\gamma} n(\gamma, t))=Q(\gamma)$,

where $n(\gamma, t)$ is the number density of electrons having a Lorentz factor $\gamma$ at a time $t$, and $\dot{\gamma} \equiv \mathrm{d} \gamma / \mathrm{d} t$ represents the radiative losses. In the case of synchrotron radiation the energy loss in cgs-units is (e.g., Rybicki \& Lightman 1979)

$\dot{\gamma}=-q B^{2} \gamma^{2}$

with

$q \equiv-\frac{2 e^{4}}{3 m_{\mathrm{e}}^{3} c^{5}}$.

Here, $B$ and $e$ are the magnetic field strength and the electron charge, respectively.

The time-independent source term $Q(\gamma)$ present in Eq. (9) gives the number of electrons injected at shocks with Lorentz factor $\gamma$ per unit of time. Relativistic electrons are injected in zones of the computational grid which separate shocked and unshocked thermal fluid. Shocks are detected using the standard criterion in the Piecewise Parabolic Method (Colella \& Woodward 1984) applied to the thermal fluid. For shock acceleration to take place, the magnetic field strength has to exceed some minimum value such that the corresponding Larmor radius $r_{\mathrm{L}}$ of the fastest particle is smaller than the smallest zone size $(\Delta L)$. This is consistent with our splitting of the spatial and temporal evolution of the non-thermal particles (see above). Therefore, the injection of electrons at shocks is limited to situations where the inequality

$\frac{r_{L}}{\Delta L}=\frac{m_{\mathrm{e}} c^{2}}{e B} \frac{\sqrt{\gamma_{\max }^{2}-1}}{\Delta L} \leq \xi$

holds. Here $\xi$ is a free parameter such that $\xi \ll 1$ (in our simulations we take $\xi=10^{-1}$ ), and $\gamma_{\max }$ is the maximum Lorentz factor of the particles which are injected into the zone (see below). In terms of the magnetic field strength, condition (10) becomes

$B \geq \frac{m_{\mathrm{e}} c^{2}}{\xi e \Delta L} \sqrt{\gamma_{\max }^{2}-1}$,

or numerically

$B \geq\left(\frac{1.7 \times 10^{3} \mathrm{~cm}}{\xi \Delta L}\right) \sqrt{\gamma_{\max }^{2}-1} \mathrm{G}$.

The injected electrons are assumed to have a power-law distribution in the interval $\left[\gamma_{\min }, \gamma_{\max }\right]$ with a power law index $p_{\text {inj }}$. Then the appropriate time-independent source term reads

$Q(\gamma)=Q_{0} \gamma^{-p_{\text {inj }}} \mathcal{S}\left(\gamma ; \gamma_{\min }, \gamma_{\max }\right)$

where $\mathcal{S}(x ; a, b)$ is the interval function:

$\mathcal{S}(x ; a, b)= \begin{cases}1 & \text { if } a \leq x \leq b \\ 0 & \text { otherwise }\end{cases}$

The magnetic field $\boldsymbol{B}$ is assumed to be randomly oriented, and its strength is parametrized by the parameter $\alpha_{B}$ which is defined as the ratio between the energy density of the magnetic field and the thermal energy density of the fluid:

$\frac{B^{2}}{8 \pi}=\alpha_{B} \frac{p}{\gamma_{\mathrm{ad}}-1}$

In our simulations the parameter $\alpha_{\mathrm{B}}$ was set equal to a value of $10^{-3}$ in order to obtain magnetic field strengths of the order of $0.1 \mathrm{G}$ in the emitting region (Bicknell \& Wagner 2002).

The emissivity $j(v)$ of the synchrotron radiation for a population of relativistic electrons with a distribution $n(\gamma)$ is given by (e.g., Rybicki \& Lightman 1979)

$j(v)=\frac{\sqrt{3} e^{3} B}{4 \pi m_{\mathrm{e}} c^{2}} \int_{1}^{\infty} \mathrm{d} \gamma n(\gamma) F\left(v / v_{0} \gamma^{2}\right)$,

where $F(x)=x \int_{x}^{\infty} \mathrm{d} y K_{5 / 3}(y) ; K_{5 / 3}(x)$ is the modified Bessel function of index $5 / 3$, and

$v_{0}=\frac{3 e B}{4 \pi m_{\mathrm{e}} c}$

In each zone of the computational grid the non-thermal electron population is represented by a sum of power laws in $N$ Lorentz factor intervals

$n(\gamma, t)=\sum_{i=1}^{N} n_{0}^{i}(t) \gamma^{-p_{i}(t)} \mathcal{S}\left(\gamma ; \gamma_{i-1}, \gamma_{i}\right)$ 
where $\gamma_{i-1}$ and $\gamma_{i}$ are the lower and upper boundaries of the $i$ th power law distribution, which at time $t$ is normalized to $n_{0}^{i}(t)$ and has a power law index $p_{i}(t)$. The $\gamma_{i}$ are logarithmically distributed according to

$\gamma_{i}=\gamma_{0}\left(\frac{\gamma_{N}}{\gamma_{0}}\right)^{(i-1) /(N-1)}, i=1, \ldots, N$

where $\gamma_{0}$ and $\gamma_{N}$ are the lower and upper limit of the whole energy interval considered for the non-thermal electron population.

Equation (9) can be solved analytically for an initial power law distribution with no injection, and for a power law injection with no initial electron distribution (Kardashev 1962). We use these solutions with a slight modification arising from the fact that we solve the equation for each energy interval separately.

The solution for the case of an initial power law distribution

$n(\gamma, 0)=n_{0} \gamma^{-p_{0}} \mathcal{S}\left(\gamma ; \gamma_{\min }, \gamma_{\max }\right)$

of index $p_{0}$ with no injection $(Q=0)$ is

$$
\begin{aligned}
n(\gamma, t)= & n_{0} \gamma^{-p_{0}}\left(1-q B^{2} \gamma t\right)^{p_{0}-2} \\
& \times \mathcal{S}\left(\gamma /\left(1-q B^{2} \gamma t\right) ; \gamma_{\min }, \gamma_{\max }\right)
\end{aligned}
$$

When no electrons are initially present $(n(\gamma, 0)=0)$, and when the injection occurs at a constant rate with a power law of index $p_{\mathrm{c}}\left(p_{\mathrm{c}}=2.2\right.$, see Sect. 2.4), i.e.,

$Q(\gamma)=Q_{0} \gamma^{-p_{\mathrm{c}}} \mathcal{S}\left(\gamma ; \gamma_{\min }, \gamma_{\max }\right)$

the solution is given by

$$
\begin{aligned}
n(\gamma, t)= & \frac{Q_{0}}{q B^{2}\left(p_{\mathrm{c}}-1\right)} \gamma^{-2}\left(\gamma_{\text {low }}^{1-p_{\mathrm{c}}}-\gamma_{\text {high }}^{1-p_{\mathrm{c}}}\right) \\
& \times \mathcal{S}\left(\gamma ; \gamma_{\min } /\left(1+q B^{2} \gamma_{\min } t\right), \gamma_{\max }\right) .
\end{aligned}
$$

$\gamma_{\text {low }}$ and $\gamma_{\text {high }}$ are evaluated according to

$$
\begin{aligned}
& \left(\gamma_{\text {low }}, \gamma_{\text {high }}\right)= \\
& \begin{cases}\left(\gamma_{\min }, \gamma_{\max }\right) & \text {;if } \frac{\gamma_{\max }}{1+q B^{2} \gamma_{\max } t} \leq \gamma<\gamma_{\min } \\
\left(\gamma_{\min }, \frac{\gamma}{1-q B^{2} \gamma t}\right) & \text {; if } \frac{\gamma_{\min }}{1+q B^{2} \gamma_{\min } t} \leq \gamma<\gamma_{\min } \\
\left(\gamma, \frac{\gamma}{1-q B^{2} \gamma t}\right) & \text {; if } \gamma_{\min } \leq \gamma \leq \frac{\gamma_{\max }}{1+q B^{2} \gamma_{\max } t} \\
\left(\gamma, \gamma_{\max }\right) & \text {; if } \frac{\gamma_{\max }}{1+q B^{2} \gamma_{\max } t}<\gamma \leq \gamma_{\max } .\end{cases}
\end{aligned}
$$

The case $\gamma_{\text {low }}=\gamma, \gamma_{\text {high }}=\gamma /\left(1-q B^{2} \gamma t\right)$ is the solution given by Kardashev (1962). This solution is also recovered in the limit of an infinite interval $\left(\gamma_{\min } \rightarrow 0, \gamma_{\max } \rightarrow \infty\right)$.

Because the kinetic equation Eq. (9) is linear in $n(\gamma, t)$, it can be solved for the distribution given in Eq. (15) using Eqs. (17) and (19) for each term in the sum separately. The new solution is then again approximated in the form of Eq. (15).

\subsection{Synchrotron radiation}

The frequency dependent synchrotron emission is computed by substituting $n(\gamma)$ from Eq. (15) into Eq. (14). Since synchrotron self-absorption is not significant in the frequency range considered $\left(10^{16}-10^{19} \mathrm{~Hz}\right)$, we compute the observed radiation by summing up the contributions from all zones of the computational grid in each time step taking into account the light travel time to the observer.

\subsection{Coupling of the thermal and non-thermal components}

In this section we explain how the energy losses of the nonthermal particles (see Sect. 2.3) are coupled to the thermal fluid. There are different ways in which $Q_{0}$ can be computed from the macroscopic hydrodynamic quantities. It is important to point out that the acceleration time scale of the electrons (Bednarz \& Ostrowski 1996) is much smaller than the hydrodynamic time scale. Therefore, following the arguments of Jones et al. (1999), we do not treat the shock acceleration process microscopically, but instead provide macroscopic models which describe the effects of the electron injection averaged over a hydrodynamic time step. Such models are not unique, and they depend on a number of free parameters, i.e., they may produce different light curves from the same hydrodynamic evolution. We will profit from this lack of uniqueness by comparing our synthetic light curves with actual observations. This will allow us to disregard injection models which do not match observed data. In the following two subsections we consider two models of electron acceleration, each with different choices of free parameters.

\subsubsection{Injection model of type-E}

Once a shock is detected, we compute $\dot{\epsilon}$, the change of the internal energy of the thermal fluid per unit of time behind shocks. We assume that $\dot{\epsilon}_{\text {acc }}$, the energy density available per unit of time to accelerate non-thermal electrons, is a fraction $\alpha_{\mathrm{e}}$ of $\dot{\epsilon}$ (Daigne \& Mochkovitch 1998; Bykov \& Meszaros 1996), i.e.,

$\dot{\epsilon}_{\mathrm{acc}}=\alpha_{\mathrm{e}} \dot{\epsilon}=\frac{\alpha_{\mathrm{e}}}{\gamma_{\mathrm{ad}}-1} \dot{p}$,

where $\dot{p}$ denotes the temporal change of the fluid pressure behind a shock due to the hydrodynamic evolution. From the definition of the source term (Eq. (13)) follows

$\dot{\epsilon}_{\mathrm{acc}}=\int_{\gamma_{\min }}^{\gamma_{\max }} \mathrm{d} \gamma \gamma m_{\mathrm{e}} c^{2} Q_{0}^{E} \gamma^{-p_{\text {inj }}}$

The back reaction of the energy loss due to particle acceleration on the flow is incorporated by decreasing the pressure in the zone(s) where the acceleration takes place during a time interval $\Delta t$. From Eq. (20) one gets

$\Delta p=-\frac{\gamma_{\mathrm{ad}}-1}{\alpha_{\mathrm{e}}} \dot{\epsilon}_{\mathrm{acc}} \Delta t$,

where $\Delta t$ is computed in the rest frame of the zone. 


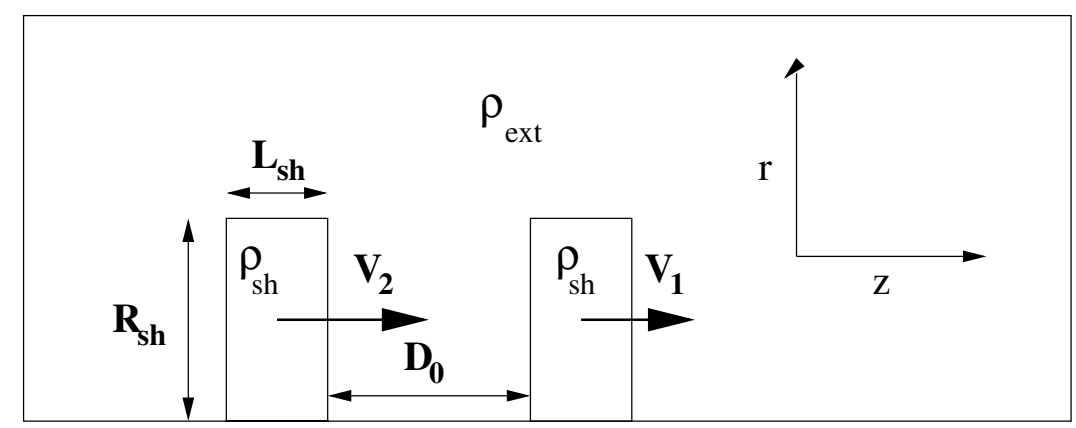

Fig. 1. A schematic view of the initial setup: two identical, cylindrical shells of radius $R_{\mathrm{sh}}$, height $L_{\mathrm{sh}}$, density $\rho_{\mathrm{sh}}$, and initial separation $D_{0}$ move through an external medium of density $\rho_{\text {ext }}<\rho_{\text {sh }}$ with velocities $V_{1}$ and $V_{2}$ along the symmetry axis $\left(z\right.$-direction) such that $V_{2}>V_{1}$.

Combining Eqs. (13), (21) and (20) one obtains

$Q_{0}^{E}=\frac{\alpha_{\mathrm{e}}\left(p_{\text {inj }}-2\right) \gamma_{\text {min }}^{p_{\text {in }}-2}}{m_{\mathrm{e}} c^{2}\left(\gamma_{\mathrm{ad}}-1\right)\left(1-\eta^{2-p_{\text {inj }}}\right)} \dot{p}$,

where $\eta \equiv \gamma_{\max } / \gamma_{\min }$.

The three free parameters of this injection model are $\alpha_{\mathrm{e}}, \eta$ and $\gamma_{\min }$, respectively.

\subsubsection{Injection model of type-N}

This model similar to the previous one in that the source term normalization $Q_{0}^{\mathrm{N}}$ satisfies the Eq. (23) but additionally the number density of electrons $n_{\mathrm{e}}^{\text {acc }}$ accelerated within a time step $\Delta t$ is parameterized to be a fraction $\zeta$ of the number of electrons in the zone

$n_{\mathrm{e}}^{\mathrm{acc}}=\zeta \frac{\rho}{m_{\mathrm{p}}}$,

where $m_{\mathrm{p}}$ is the proton mass. Using Eq. (13) one finds

$n_{\mathrm{e}}^{\mathrm{acc}}=\Delta t \frac{Q_{0}^{N}}{p_{\text {inj }}} \gamma_{\text {min }}^{1-p_{\text {inj }}}\left(1-\eta^{1-p_{\text {inj }}}\right)$.

Using Eqs. (23)-(25) one obtains

$\gamma_{\text {min }}=\frac{p_{\text {inj }}-1}{p_{\text {inj }}-2} \frac{\alpha_{\mathrm{e}}}{\zeta} \frac{1-\eta^{1-p_{\text {inj }}}}{1-\eta^{2-p_{\text {inj }}}} \frac{m_{\mathrm{p}}}{m_{\mathrm{e}} c^{2}} \frac{\dot{p} \Delta t}{\left(\gamma_{\mathrm{ad}}-1\right) \rho}$,

for the minimum Lorentz factor, and

$Q_{0}^{N}=\frac{\zeta}{m_{\mathrm{p}}} \frac{\rho}{\Delta t} \frac{p_{\mathrm{inj}}-1}{\gamma_{\min }^{1-p_{\text {inj }}}\left(1-\eta^{1-p_{\text {inj }}}\right)}$,

for the source normalization, where $\gamma_{\min }$ is given by Eq. (26).

The three free parameters of this injection model are $\alpha_{\mathrm{e}}, \eta$, and $\zeta$, respectively.

\section{Results}

\subsection{Hydrodynamic setup}

The density of the two colliding, identical shells is $\rho_{\mathrm{sh}}=$ $10^{4} \rho_{\mathrm{ext}}=10^{-22} \mathrm{~g} \mathrm{~cm}^{-3}$, and their temperature is half the temperature of the external medium $T_{\mathrm{ext}}=7 \times 10^{7} \mathrm{~K}$. The two shells move with Lorentz factors $\Gamma_{1}=3$ and $\Gamma_{2}=15$, respectively. Initially, both shells are of cylindrical shape with a height $L_{\mathrm{sh}}=10^{14} \mathrm{~cm}$ and a radius $R_{\mathrm{sh}}=10^{16} \mathrm{~cm}$. The shells are initially separated by a distance $D_{0}=5 \times 10^{14} \mathrm{~cm}$. The initial setup is shown schematically in Fig. 1.

The two colliding shells modeled in our simulations are initially located near the left boundary of the computational grid at $z=z_{\min }$ (Sect. 1). When the leading of the two shells approaches the right boundary of the grid at $z=z_{\max }$, the grid is translated into positive $z$-direction such that both shells are again located near the left boundary of the grid at $z=z_{\min }$. In order to prevent any numerical artifact due to this re-mapping close to the left grid boundary at $z_{\min }$, we place the shells sufficiently far from that boundary such that the Riemann structure emerging from the back of the trailing shell remains practically unaffected.

The hydrodynamic set up consists of a two-dimensional computational grid in cylindrical coordinates $(r, z)$ of $40 \times$ 2000 zones covering a physical domain of $1.5 \times 10^{16} \mathrm{~cm}$ by $5 \times 10^{15} \mathrm{~cm}$. The grid is initially filled with an external medium at rest, which has a uniform density $\rho_{\mathrm{ext}}=10^{-26} \mathrm{~g} \mathrm{~cm}^{-3}$ and a uniform pressure $p_{\mathrm{ext}}=10^{-11} \mathrm{erg} \mathrm{cm}^{-3}$. After every remapping the computational domain ahead of the shells is refilled with that external medium.

We point out that the ratio $\chi=\rho c^{2} / 4 p$ is rather large (according to Bicknell \& Wagner 2002) in the shells initially $\left(\chi \approx 4.5 \times 10^{4}\right)$, but it decreases considerably during the hydrodynamic evolution (see next section).

\subsection{Hydrodynamic evolution}

As the shells are set up as sharp discontinuities in a uniform external medium they experience some hydrodynamic evolution before the actual collision, which starts when the Riemann structure trailing the slower leading shell meets the bow shock of the fast trailing shell. This pre-collision evolution is rather similar for both shells, and can be estimated analytically using an exact one dimensional Riemann solver. In Fig. 2 the two top panels show the analytic evolution of the flow conditions. The front (with respect to the direction of motion) discontinuity of each shells decays into a bow shock ( $S 1 b$ and $S 2 b)$, a contact discontinuity (in Fig. 2 we only show a zoom of the one corresponding to the leading shell, $C D 1 R$ ), and a reverse shock ( $S 1 a$ and $S 2 a$ ). The back discontinuity of each shells develops into a rarefaction $(R 1 b$ and $R 2 b)$ that connects the still 


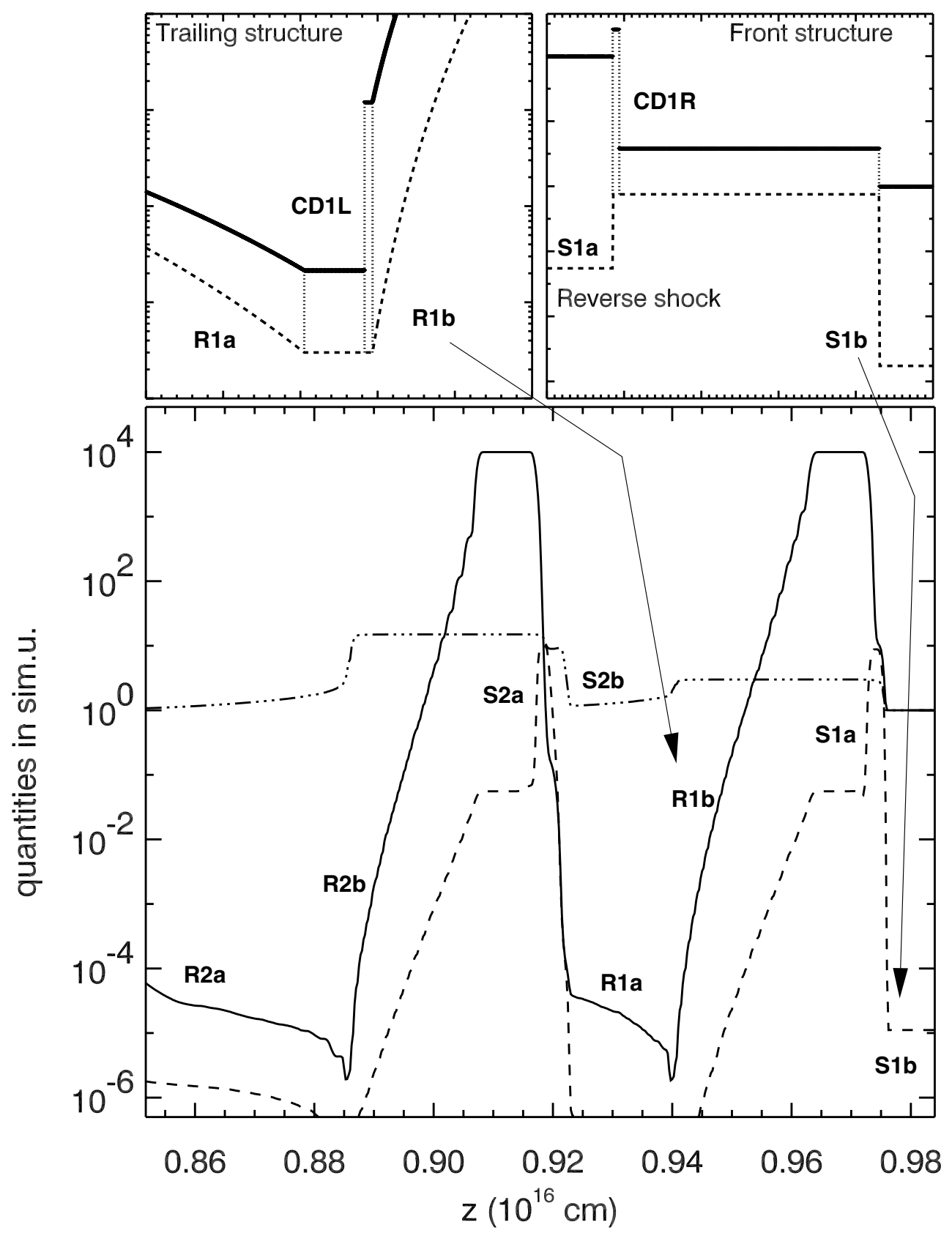

Fig. 2. Snapshot illustrating the flow structure along the symmetry axis arising from the setup described in Fig. 1 just before the two shells start to interact $\left(t=27 \mathrm{ks}\right.$ ). The lower panel shows the density (solid line) and pressure (dashed line) distribution measured in units of $\rho_{\mathrm{ext}}$ and $\rho_{\mathrm{ext}} c^{2}$, respectively. The dash-dotted line gives the Lorentz factor of the fluid which is moving towards the right. The upper left (right) panel displays the exact solution of the one dimensional Riemann problem defined by the trailing (leading) edge of the right shell. Labeled are the two bow shocks $S 1 b$ and $S 2 b$, the two reverse shocks $S 1 a$ and $S 2 a$, the four rarefactions $R 1 a, R 1 b, R 2 a$ and $R 2 b$, and (in the top panels only) the contact discontinuities $C D 1 L$ and $C D 1 R$.

unperturbed state inside the shell with a contact discontinuity separating shell matter from the external medium (in Fig. 2 only the leading shell is labelled, $C D 1 L$ ), and into a second rarefaction that connects the contact discontinuity with the external medium $(R 1 a$ and $R 2 a)$.

The pre-collision evolution is qualitatively similar when instead of sharp discontinuities a more smooth transition between the shells and the external medium is assumed. The Riemann structure emerging from the edges of the shells will be the same, i.e., it will consists of the same structure of shocks and rarefactions as with our set up. However, the exact values of the state variables in the intermediate states connecting the conditions in the shells with the external medium will be obviously different.

The pre-collision hydrodynamics has two direct consequences. Firstly, each shell is heated by a reverse shock $(S 1 a$ and $S 2 a$ ) which increases $\chi$ by about two orders of magnitude (Fig. 2). Secondly, both shells are spread in $z$ direction as 

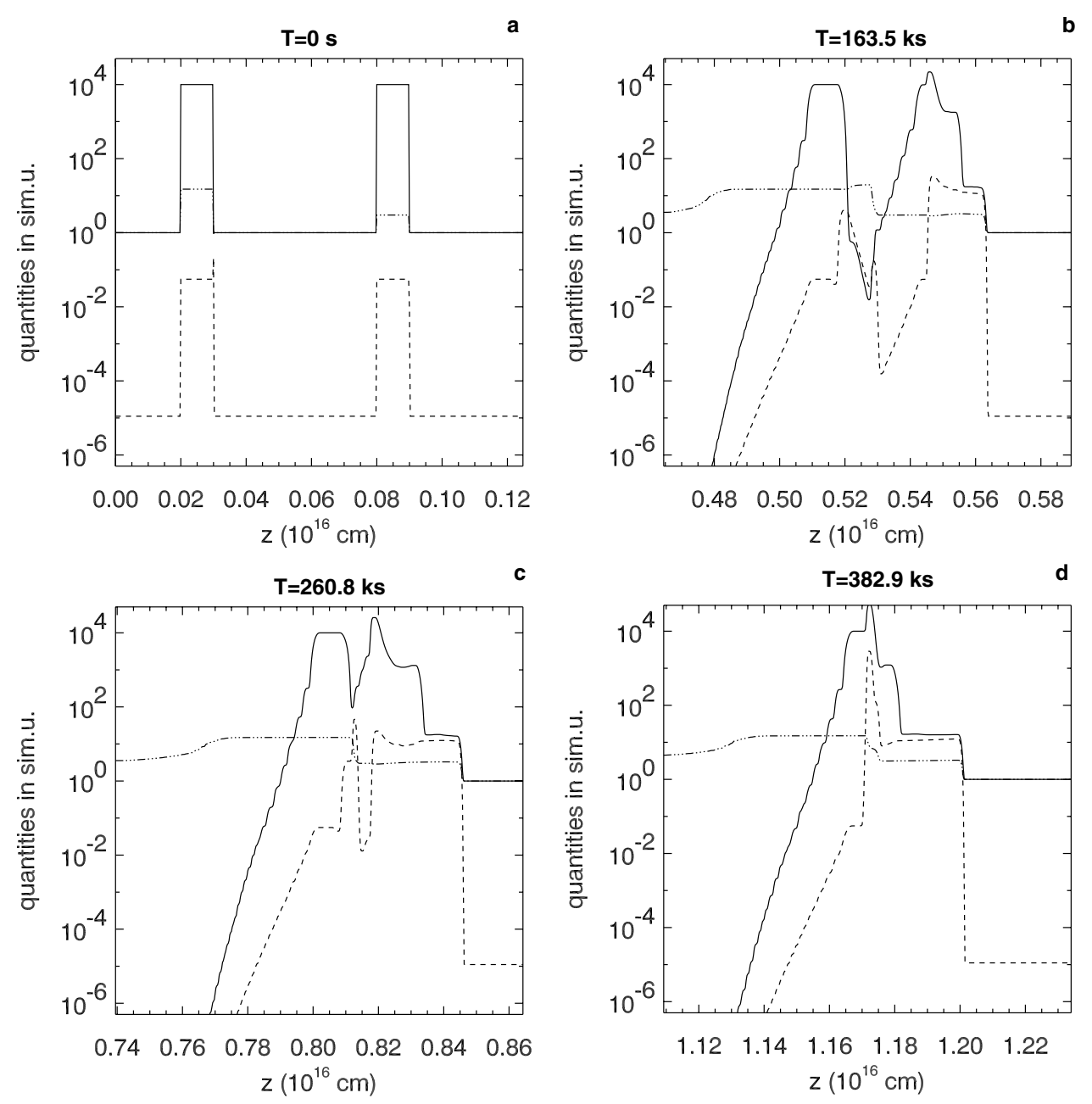

Fig. 3. Snapshots of the rest mass density (solid line; in units of $10^{-26} \mathrm{~g} \mathrm{~cm}^{-3}$ ), pressure (dashed line; in units of $10^{-5} \mathrm{erg} \mathrm{cm}^{-3}$ ), and Lorentz factor of the fluid (dash-dotted line) along the symmetry axis of the computational grid at four different stages of the evolution.

external medium shocked in the bow shocks $(S 1 b$ and $S 2 b)$ piles up in front of the shells. The latter effect is complicated in case of the faster trailing shell by the fact that its bow shock $(S 2 b)$ soon starts to interact with the rarefaction $(R 1 a)$ of the slower leading shell. Thereby the bow shock speeds up, and it eventually catches up with the slower leading shell. Our simulations show that the resulting interaction of the two shells occurs at a distance which is slightly smaller than the distance derived from an analytic estimate (see below). The accelerating bow shock $S 2 b$ drags along the whole Riemann structure. This explains why the state behind $S 2 b$ is not uniform (as in case of the slower leading shell), but shows a monotonically decreasing density and pressure distribution (Fig. 2). It further explains why the density behind the reverse shock of the faster shell $(S 2 a)$ is always less than that behind the reverse shock $(S 1 a)$ of the slower shell.

Before the bow shock $S 2 b$ of the faster trailing shell can enter the interior of the slower shell where $\rho=\rho_{\mathrm{sh}}$, it has to cross the rarefaction $R 1 b$, i.e., it has to propagate through a steadily increasing density. Hence, the emission produced by the shock will increases gradually during this epoch until it becomes an internal shock propagating through the slower shell (Figs. 3 and 4). We point out that in the analytic model the internal shock does appear instantaneously when the two shells touch each other.

Using our initial conditions (see previous section) an analytic estimate of the time when the two shells collide is given by

$$
\begin{aligned}
T_{\mathrm{c}}^{\mathrm{an}} & =\frac{D_{0}}{V_{2}-V_{1}} \\
& =\frac{D_{0}}{c}\left(\sqrt{1-\Gamma_{2}^{-2}}-\sqrt{1-\Gamma_{1}^{-2}}\right)^{-1}=280.7 \mathrm{ks} .
\end{aligned}
$$

From our simulation we find that $T_{10}=0.913 T_{\mathrm{c}}^{\mathrm{an}}, T_{50}=$ $0.977 T_{\mathrm{c}}^{\mathrm{an}}$ and $T_{90}=1.009 T_{\mathrm{c}}^{\mathrm{an}}$, where $T_{10}, T_{50}$ and $T_{90}$ are three moments of time at which the minimum density ahead of the faster shell is $10 \%, 50 \%$ and $90 \%$ of the instantaneous maximum density $\rho_{\max }$ on the faster shell. Note that $\rho_{\max }$ does not coincide, in general, with the initial density of the faster shell because the shell has undergone some hydrodynamic evolution. However, $\rho_{\max }$ is only a few per cent larger than $\rho_{\text {sh }}$ for the models under consideration.

An analytic estimate of the time at which an observer located at a distance $c t_{0}$ from the initial position of the faster shell will receive the first light is

$T_{\mathrm{arr}}^{\mathrm{an}}=T_{\mathrm{c}}^{\mathrm{an}}\left(1-V_{2} / c\right)+t_{0}$. 


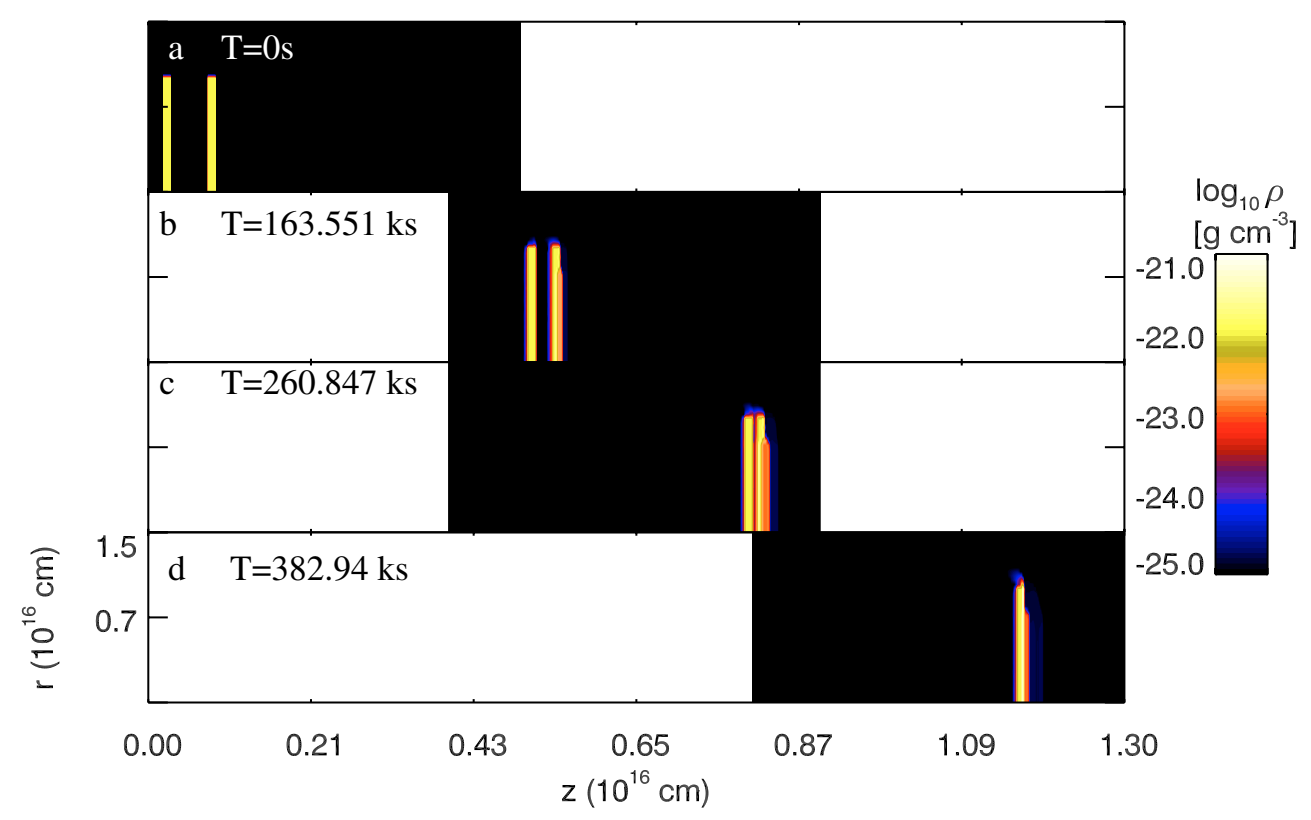

Fig. 4. Contour plots of the logarithm of the rest mass density at the same moments of time as in Fig. 3. The coordinate values at both the $r$ and $z$-axis are in units of $10^{16} \mathrm{~cm}$. The grid re-mapping procedure (see Sect. 3.1) is the cause for the shift of the computational domain seen in panels b)-d).

As mentioned in the previous section, the ratio $\chi$ decreases during the interaction by almost four orders of magnitude because of the large pressure increase (from its initial value $\approx 10^{4}$; Fig. 3 panel), while the density only increases by a factor of $\approx 2$. We note that the interaction region is the only one which produces a significant amount of all observed radiation. Hence, the value of $\chi$ in that region is the one relevant for observations and not the initial one.

\subsection{Setup of the non-thermal component}

The non-thermal electrons are binned into $N=48$ logarithmically spaced intervals covering a total Lorentz factor interval $\left[\gamma_{1}=1, \gamma_{N}=10^{8}\right]$ (Sect. 2.2). The source term describing the electron injection at shocks (Sect. 2.4) has a power law in$\operatorname{dex} p_{\text {inj }}=2.2$, which is compatible with the value found by Bednarz \& Ostrowski (1998). The injection source terms are computed for the model type-E (Sect. 2.4.1) with the parameters $\alpha_{\mathrm{e}}=10^{-2}, \eta=10^{4}$ and $\gamma_{\min }=50$, and for the model type-N (Sect. 2.4.2) with the parameters $\alpha_{\mathrm{e}}=10^{-2}, \eta=10^{4}$ and $\zeta=10^{-2}$, respectively.

In order to produce light curves, the synchrotron emissivity in each radiating zone is computed in 24 logarithmically spaced frequency bands ranging from $10^{16} \mathrm{~Hz}$ to $10^{19} \mathrm{~Hz}$. In a subsequent step a soft and a hard light curve is obtained by integrating the computed synchrotron spectra over a prescribed energy interval. For the soft light curve we considered photon energies between 0.1 and $1 \mathrm{keV}$, and for the hard light curve energies between 2 and $10 \mathrm{keV}$.

\subsection{Evolution of the non-thermal component}

Figure 5 shows the normalized soft and hard X-ray light curves computed from the hydrodynamic evolution using the

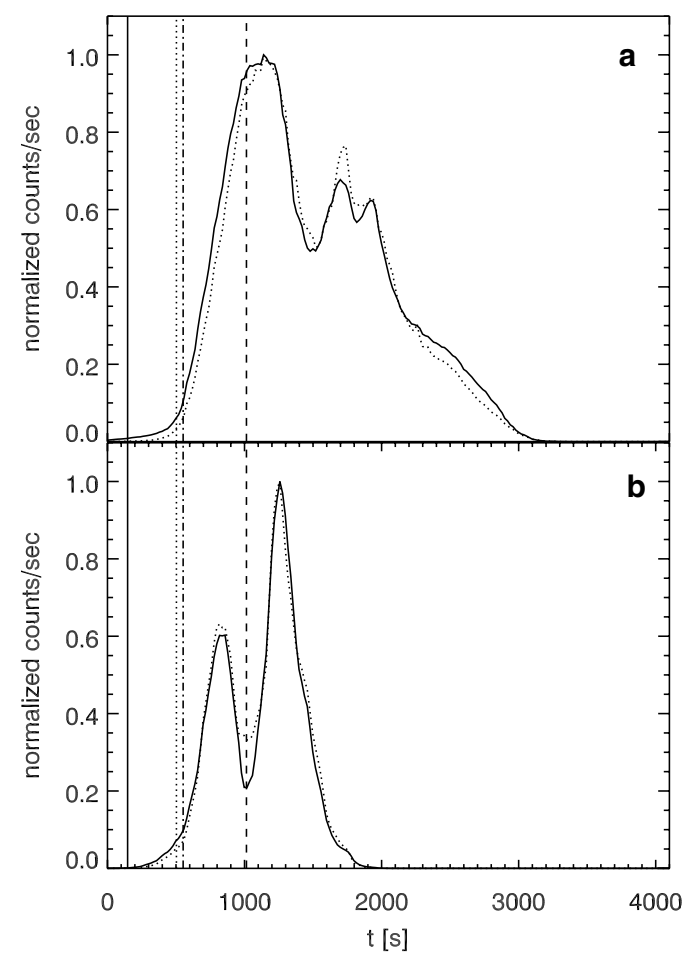

Fig. 5. Normalized soft (solid line) and hard (dotted line) X-ray light curves in the observer's frame for injection of type-E a) and type-N b), respectively. The light curves are binned into $20 \mathrm{~s}$ time bins. The vertical lines correspond to $T_{10}$ (solid), $T_{50}$ (dotted), $T_{90}$ (dashed), and $T_{\text {arr }}^{\text {an }}$ (dash-dotted), respectively (for a definition of these times see Sect. 3.2). Note that the time coordinate is renormalized to the time at which the count rate first exceeds $10^{-3}$ of the count rate at maximum.

procedure described in the previous section. Because the shells start to interact earlier than predicted by commonly used analytic estimates (see, e.g., Spada et al. 2001) the first peak of 


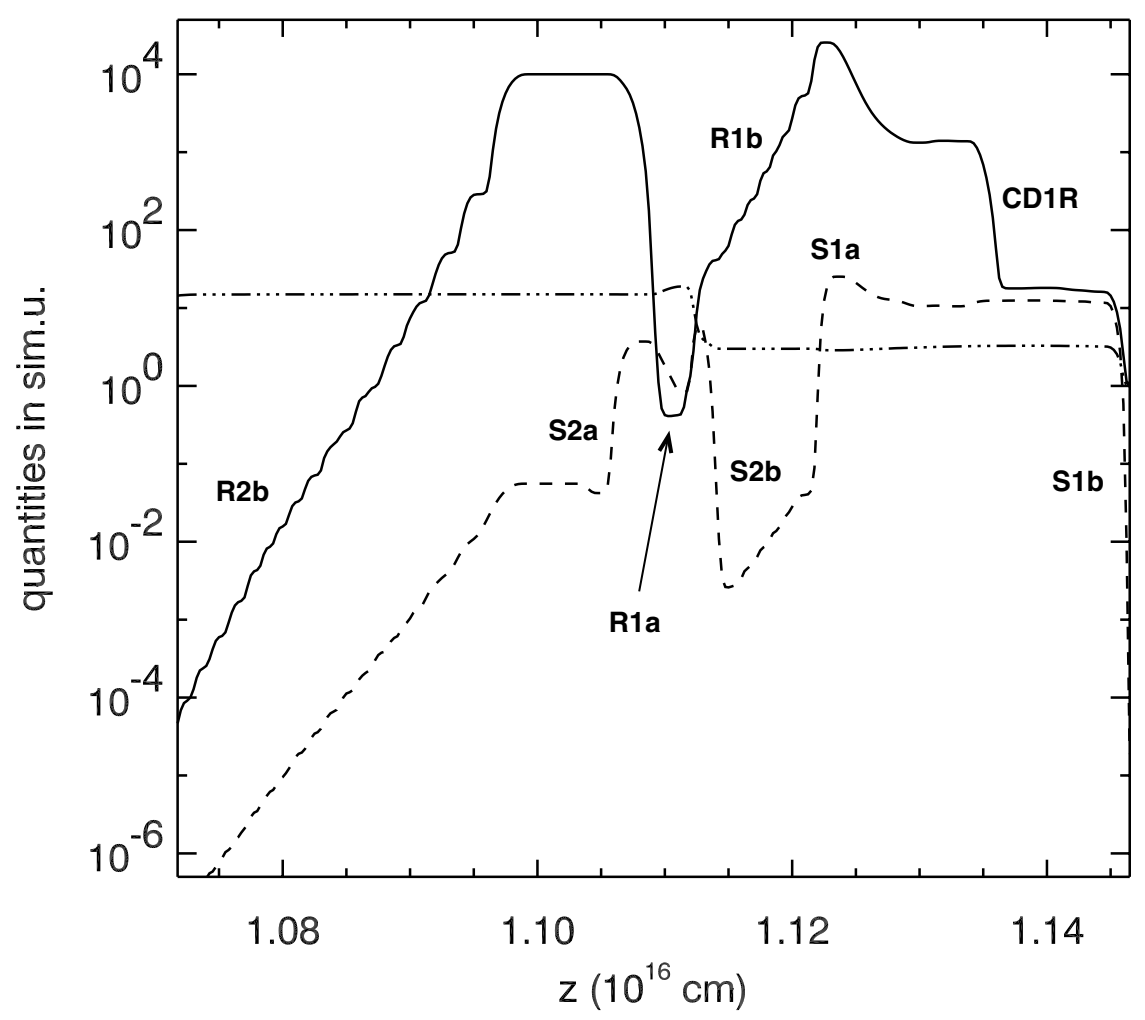

Fig. 6. Snapshot illustrating the flow structure along the symmetry axis after the two shells start to interact $(t=212 \mathrm{ks})$. The figure shows the density (solid line) and pressure (dashed line) distribution measured in units of $\rho_{\mathrm{ext}}$ and $\rho_{\mathrm{ext}} c^{2}$, respectively. The dash-dotted line gives the Lorentz factor of the fluid which is moving towards the right. Labeled are the two bow shocks $S 1 b$ and $S 2 b$, the two reverse shocks $S 1 a$ and $S 2 a$, the rarefactions $R 1 a, R 1 b$ and $R 2 b$, and the contact discontinuity $C D 1 R$. At this time the Riemann structure emerging from the leading edge of the faster shell is interacting with the trailing Riemann structure from the rear edge of the slower shell. This leads to the effective disappearance of the contact discontinuity $C D 1 L$ and to the interchange of the position of $R 1 a$ and $S 2 b$.

the light curve is observed well before the time $T_{90}$ defined at the end of Sect. 3.2 (Fig. 5, panel b) for the injection model of type-N. Such an effect is not present in the injection model of type-E. The analytically predicted arrival time of the radiation $T_{\text {arr }}^{\text {an }}$ (Eq. (29)) should provide a good marker for the onset of the light curve. However, $T_{\text {arr }}^{\text {an }}$ depends on the exact location of the emitting zones and on the specific injection model used. In the simulated collisions the spatial arrangement of the emitting zones (shocks) is different from that of non-evolving shells (analytic case), because some hydrodynamic evolution already takes place before the shells directly interact. The arrival time computed from our simulations is hence smaller than $T_{\text {arr }}^{\text {an }}$.

Our simulations show that the double peak structure of the light curves is caused by the bow shock $S 2 b$ propagating into the slower leading shell, and by the reverse shock $S 2 a$ propagating through the faster trailing shell (see Fig. 2). Both of these two shocks are boosted after the actual shell interaction starts. We further find that the relative height of the light curve peaks depends on the injection model. In case of the model type-E (Fig. 5, panela) the first light curve peak caused by the (former) bow shock $S 2 b$ is higher than the second peak caused by the reverse shock $S 2 a$, because the rate of change of the energy density per unit of volume in shock $S 2 b$ is found to be larger than in the reverse shock $S 2 a$. Hence, shock $S 2 b$ provides a larger amount of dissipation than shock $S 2 a$ (see Eq. (20)), i.e., more electrons are accelerated in the shock $S 2 b$ than in the shock $S 2 a$. In case of the model type-N (Fig. 5, panel b) we find that less electrons are accelerated in the shock $S 2 b$ than in the case of model type-E, because their number density, which is proportional to the fluid density (Eq. (24)), is smaller in the (former) bow shock $S 2 b$ than in the reverse shock $S 2 a$ (see Fig. 6).

Both light curves are binned into 20s time bins. Thus, if the temporal resolution of an observations is much worse (effectively it is $\simeq 80 \mathrm{~s}$ due to signal-to-noise ratio limitations; Brinkmann et al. 2003), the finest time structures of our computed light curves will partially or even completely be smeared out, i.e., the differences between the two injection models could not be resolved.

\subsection{Energy}

Initially, the kinetic $\left(E_{\mathrm{k}}^{\mathrm{ini}}\right)$ and thermal $\left(E_{\mathrm{th}}^{\mathrm{ini}}\right)$ energies of the two shells are

$E_{\mathrm{k}}^{\mathrm{ini}}=L_{\mathrm{sh}} R_{\mathrm{sh}}^{2} \pi \rho_{\mathrm{sh}}\left(\Gamma_{1}+\Gamma_{2}-2\right) c^{2}=4.4 \times 10^{46} \mathrm{erg}$

and

$E_{\mathrm{th}}^{\mathrm{ini}}=L_{\mathrm{sh}} R_{\mathrm{sh}}^{2} \pi \frac{p_{\mathrm{sh}}}{\gamma_{\mathrm{ad}}-1}=4.7 \times 10^{40} \mathrm{erg}=9.4 \times 10^{-7} E_{\mathrm{k}}^{\mathrm{ini}}$ 
respectively. Beyond the time $(t=382.9 \mathrm{ks})$ at which no radiation is being produced anymore, the kinetic and internal energies of the fluid are

$E_{\mathrm{k}}^{\mathrm{fin}}=4.35 \times 10^{46} \mathrm{erg}=0.99 E_{\mathrm{k}}^{\mathrm{ini}}$,

and

$E_{\mathrm{th}}^{\mathrm{fin}}=4.4 \times 10^{44} \mathrm{erg}=0.01 E_{\mathrm{k}}^{\mathrm{ini}}$,

respectively. The total radiated energy obtained by integrating the light curve derived from the integrated synchrotron spectra between 0.1 and $10 \mathrm{keV}$ in time is (using model type-N):

$E_{\mathrm{rad}}^{\mathrm{X}}=1.4 \times 10^{43} \mathrm{erg}=0.03 E_{\mathrm{Th}}^{\mathrm{fin}}$

with a peak luminosity of $5.8 \times 10^{39} \mathrm{erg} \mathrm{s}^{-1}$. Therefore, the efficiency of converting thermal energy into $\mathrm{X}$-ray radiation is $E_{\text {rad }}^{\mathrm{X}} / E_{\mathrm{th}}^{\mathrm{fin}} \simeq 0.03$.

We notice that after the collision the resulting merged shell is much hotter than the initial two shells $\left(E_{\mathrm{th}}^{\mathrm{fin}} \gg E_{\mathrm{th}}^{\mathrm{ini}}\right)$. This heating caused by the internal shocks, and by the pre-collision hydrodynamic evolution of the shells.

\section{Discussion}

Many of the effects found in our axisymmetric simulations can also be quite accurately modeled assuming a one-dimensional hydrodynamic evolution. Indeed, the initial set-up is explicitly chosen to match this one-dimensionality, because we want to compare our simulation results with those obtained from previous analytic one-zone $1 \mathrm{D}$ models. However, we stress that 1D models represent only a first and rough attempt to model the physics of blazars, which have a multidimensional nature. In the previous sections we have demonstrated that our simulation results match qualitatively with those of previous 1D models. Thus, we are able to explore different initial configurations where multidimensional effects are expected to play a more important role (e.g., interaction of shells of different shell radii, inhomogeneities in the external medium, etc.).

\subsection{Shell interaction}

Concerning the temperature of the shells, which is chosen to be half the temperature of the external medium (Sect. 3.1), we do not expect any significant change of the dynamic evolution or the non-thermal emission, as long as density and pressure contrast between the shells and the external medium is sufficiently large, and as long as the temperature within the shells does not greatly exceed that of the ambient medium. If the latter requirement were to be violated, which is not expected according to observational data, the shells would emit a significant amount of radiation before they collide.

A qualitatively similar shell evolution and shell interaction is to be expected if instead of two perfectly (sharp) cylindrical shells one were to consider shells having a Gaussian (smooth) distribution of density, pressure, and Lorentz factor. Of course, the exact values of the arrival time, the time of interaction, etc., would be slightly different in this case. Decreasing the density, pressure, or Lorentz factor contrast between the shell and the external medium will decrease the speed of propagation of the Riemann structures emerging both from the leading and trailing edges (normal to the direction of propagation) of the shell. Hence, the collision time would be even closer to the analytic estimate, and the shells would experience less pre-collision hydrodynamic evolution (Sect. 3.2).

It would be particularly interesting to study the influence of a moving external medium mimicking the flow of an underlying rarefied jet. In this situation the Lorentz factor contrast between the shells and the external medium can be substantially smaller (even close to zero, if the jet moves almost as fast as the slower shell). Thus, the size and the depth of the Riemann structures produced by the shells will be much smaller. This has the important consequence that the effect of the rarefactions trailing the slower shell on the leading edge of the faster shell will be reduced.

Decreasing the velocity difference between the two shells prolongs the pre-collision epoch, and reduces the strength of the internal shocks. Under these circumstances one expects that a smaller amount of energy is emitted in the X-rays bands.

\subsection{Shock acceleration models}

The time scale $\tau_{\text {acc }}$ of the shock acceleration process $\left(\tau_{\text {acc }} \approx\right.$ $0.2 \mathrm{~s}$ for, e.g., $B=0.1 \mathrm{G}$ and $\gamma=5 \times 10^{5}$; see Sect. 2.4 ) is much shorter than the typical hydrodynamic time step $\Delta t$ of the simulation $(\Delta t \approx 15 \mathrm{~s})$. Therefore, we have to parameterize the effect of the shock acceleration process on time scales of the order of the hydrodynamic time scale.

The two different parameterizations of the shock acceleration process discussed in Sect. 2.4 produce qualitatively different light curves. An increase of the fraction of dissipated energy available for the acceleration of electrons $\left(\alpha_{\mathrm{e}}\right)$ produces either more electrons (injection model type-E; Sect. 2.4.1) or injects electrons at higher energies (injection model type-N; Sect. 2.4.2). Increasing the ratio between the upper and lower Lorentz factor limit of the injection interval $\left(\eta \equiv \gamma_{\max } / \gamma_{\min }\right)$ produces more electrons at higher energies. Our particular choice of parameters is constrained by the fact that the maximum of the X-ray synchrotron emission is assumed to occur around $10^{16}-10^{17} \mathrm{~Hz}$. The particular choice of the parameter $\zeta$ (the fraction of electrons accelerated in a zone within a hydrodynamic time step; Sect. 2.4.2) is suggested by fits of observed blazar spectra which imply electron number densities in the range of $10^{3}-10^{4} \mathrm{~cm}^{-3}$ (Maraschi et al. 2003).

\subsection{Efficiency}

The particular shell collision which we have simulated has an efficiency of the order of $1 \%$ in converting thermal energy to $\mathrm{X}$-ray radiation (see Sect. 3.5). However, if the flow contains more than two shells at the same time, the total efficiency of converting thermal to radiation energy might be larger. 


\subsection{Light curves}

The typical time scale of the flare in the observer's frame is found to be of the order of one kilosecond (Fig. 5). However this finding could be influenced by our particular choice of the shell width and the magnetic field strength. The shape of the light curve will depend on the relation between the time scales associated with the radiation processes and the physical size of the shells (Mastichiadis \& Kirl 2002). On the one hand, the synchrotron cooling time scale $\tau_{\mathrm{c}}$ of the non-thermal electrons in the comoving frame of the shell is $\tau_{\mathrm{c}}=\left(q B^{2} \gamma\right)^{-1}=1.6 \times$ $10^{4} \mathrm{~s}$ for $B=0.25 \mathrm{G}$ and $\gamma=5 \times 10^{5}$ (see Sect. 2.2). On the other hand, this time scale is comparable to the light crossing time of the shell in the same frame $\left(T_{\mathrm{LC}}=L_{\mathrm{sh}} / c \Gamma \simeq 3.4 \times 10^{4} \mathrm{~s}\right.$; see Sect. 3.1). Thus, electrons have sufficient time to radiate away a large fraction of their energy before they leave the shell.

The light curves show a fast-rise slow-decline structure in both the soft and the hard energy band (Fig. 5). It can be recognized that in the shell collision considered in our simulation no significant time delays are observed between the hard and soft X-rays.

We have checked that the patterns displayed by, at least, the hard light curves (Fig. 5) are similar to the ones found by Brinkmann et al. (2003) for Mrk 421 in their Figs. 6 and 9 (corresponding to different orbits of $X M M$ ), respectively. We point out that the time scales of the observed light curves of Mrk 421 are several times longer than ours. However, this does not invalidate our comparison, because the duration of the synthetic light curves depends on the exact physical size of the shells (the wider they are in the $z$-direction, the longer the duration).

\section{Conclusions}

We have performed relativistic hydrodynamic simulations of dense shells of plasma moving in a rarefied medium, and computed the X-ray light curves produced during their collision. Our simulations improve existing analytic (one-zone) models (e.g., Spada et al. 2001) by computing a more realistic hydrodynamic evolution of the fluid.

The physical conditions in the external medium correspond to the standard jet conditions in blazars (Takahashi et al. 2000), while those of the shells, in particular the density contrast between the shells and the external medium, are in agreement with the estimates of Bicknell \& Wagner (2002). These two facts enable us to properly address the physics of internal shocks in blazars (in contrast to what is claimed in Bicknell \& Wagner 2002). Indeed, we have demonstrated the ability of our method to produce synthetic light curves from relativistic hydrodynamic simulations including the back reaction of the emitted radiation on the thermal fluid. We find that both the total radiated energy and the light curves agree well with observational data. With our method the total efficiency of conversion of thermal energy into radiation energy need not be assumed, but can be directly computed. For the physical parameters used in our simulations we obtain a total efficiency of about $1 \%$.
Our results show that the detailed structure of the synthetic light curves depends on the particular choice of the macroscopic parameterization of the process of particle acceleration. By comparing our results with observed light curves of Mrk 421 we find that both models of particle acceleration used in our simulations provide light curve patterns that can be identified in observed light curves. However, it seems that our injection model type-E (where a prescribed fraction of the change of the internal energy of the thermal fluid per unit time is used to accelerate non-thermal electrons) fits both the soft and the hard light curves equally well, while the injection model type-N (similar to type-E, but where only a prescribed fraction of the available electrons is accelerated) fits only the hard part of the light curve. The different results obtained with the two models of injection are related to the fact that while the injection model type-E accounts for the strength of the shocks (the number of particles injected is proportional to the rate of change of energy per unit of volume behind the shock), the model type- $\mathrm{N}$ does not.

The light curve produced by a single collision of two shells does not necessarily produce a single bumped flare, but can consist of several peaks as our simulations demonstrate. These peaks result from shocks which form after the actual interaction starts. One of these shocks propagates into the slower leading shell, while the second shock propagates into the faster trailing shell. This behavior is clearly different from that of one-zone models, which only predict a single peak from a single interaction. A comparison of our results with observed light curves of Mrk 421 shows that some of the observed flares display a multiple peak structure that can be well accommodated by our model. As a further consequence we note that our model can explain variability in the light curves with fewer components (shells) than one-zone models.

The results presented in this paper represent the beginning of a set of studies where we will consider different initial configurations to study the influence of such different initial conditions on the light curves of blazars. Among the possibilities arising we can immediately include more shells in the simulations, or we can vary the size of the shells. The latter variation, particularly, will allow us to include multidimensional effects by considering shells of different radial size. A further improvement of the method will be to include inverse Compton processes in the treatment of the radiation, which is of particular relevance in the study of blazars.

The present approach relies on the assumption that the magnetic field is dynamically negligible, randomly oriented, and its energy density is proportional to the thermal pressure. Thus, another step forward is to include dynamically important magnetic fields in the simulations. Finally, we also consider the possibility of performing 3D simulations which are better adapted to the configurations expected in nature (e.g., non-aligned shells, multidimensional shell trajectories, genuine 3D shell shapes, etc.). In fact both GENESIS and the newly coded radiative part are written (and tested) for 3D applications. However, due to the huge amount of computational power required for a $3 \mathrm{D}$ simulation, we have not performed such simulations yet. 
Acknowledgements. M.A.A. acknowledges the EU-Commission for a fellowship (MCFI-2000-00504). P.M. acknowledges support by the International Max-Planck Research School on Astrophysics (IMPRS). We are very grateful to Francesco Miniati for the careful reading of this manuscript and his useful comments.

\section{References}

Aloy, M. A., Ibáñez, J. M., Martí, J. M., \& Müller, E. 1999, ApJS, 122,151

Bednarz, J., \& Ostrowski, M. 1998, Phys. Rev. Lett., 80, 3911

Bednarz, J., \& Ostrowski, M. 1996, MNRAS, 283, 447

Bicknell, G. V., \& Wagner, S. J. 2002, PASA, 19, 192

Bregman, J., Maraschi, L., \& Urry, C. M. 1987, in Exploring the Universe with the IUE Satellite, ed. Y. Kondo (Dordrecht: Reidel), 685

Brinkmann, W., Sembay, S., Griffiths, R. G., et al. 2001, A\&A, 365, L162

Brinkmann, W., Papadakis, I. E., den Herder, J. W. A., \& Haberl, F. 2003, A\&A, 402, 929

Bykov, A. M., \& Meszaros, P. 1996, ApJ, 461, L37

Colella, P., \& Woodward, P. R. 1984, J. Compt. Phys., 54, 174

Daigne, F., \& Mochkovitch, R. 1998, MNRAS, 196, 275

Edelson, R., Griffiths, G., Markowitz, A., et al. 2001, ApJ, 554, 274

Fossati, G., Celotti, A., Chiaberge, M., et al. 2000, ApJ, 541, 153

Jones, T. W., Ryu, D., \& Engel, A. 1999, ApJ, 512, 105
Kardashev, N. S. 1962, Sov. Astr., 6, 317

Kataoka, J., Takahasi, T., Makino, F., et al. 2000, ApJ, 528, 243

Kataoka, J., Takahashi, T., Wagner, S. J., et al. 2001, ApJ, 560, 569

Malizia, A., Capalbi, M., Fiore, F., et al. 2000, MNRAS, 312, 123

Maraschi, L., \& Tavecchio, F. 2003, ApJ, 593, 667

Martí, J. M., Müller, E., \& Ibáñez, J. M. 1994, A\&A, 281, L9

Mastichiadis, A., \& Kirk, J. G. 2002, PASA, 19, 138

Miniati, F. 2001, CPC, 141, 17

Moderski, R., Sikora, M., \& Blazejowski, M. 2003, A\&A, 406, 855

Pian, E. 2002, Publ. Astr. Soc. Austr., 19, 49

Sikora, M., Blazejowski, M., Begelman, M. C., \& Moderski, M. 2001, ApJ, 561, 1154

Rybicki, G. B., \& Lightman, A. P. 1979, Radiative Processes in Astrophyisics (New York: Wiley Interscience Publication)

Spada, M., Ghisellini, G., Lazzatti, D., \& Celotti, A. 2001, MNRAS, 325,1559

Takahashi, T., Tashiro, M., Madejski, G., et al. 1996, ApJ, 470, L89

Takahashi, T., Kataoka, J., Madejski, G., et al. 2000, ApJ, 542, L105

Tanihata, C. 2002, Ph.D. Thesis Tokyo Univ., ISAS Research Note, 739

Tanihata, C., Takahashi, T., Kataoka, J., \& Madejski, G. M. 2003, ApJ, 584, 153

Tanihata, C., Urry, C. M., \& Takahashi, T., et al. 2001, ApJ, 563, 569

Urry, C. M., \& Padovani, P. 1995, PASP, 107, 803

Zhang, Y. H., Celotti, A., Treves, A., et al. 1999, ApJ, 527, 719 